Raphael Câmara Medeiros Parente ${ }^{1}$

VILMON DE FREITAS ${ }^{2}$

Rodrigo SOARES DE MOURA NETO ${ }^{3}$

Marco Aurélio Pinho de Olivelra ${ }^{4}$

RICARDO BASSIL LASMAR ${ }^{5}$

Marisa Teresinha Patriarca ${ }^{6}$

FeliPe Simões CANAVEZ ${ }^{7}$

\section{Metaplasia óssea endometrial: quadro clínico e seguimento após tratamento}

\author{
Endometrial osseous metaplasia: Clinical presentation and follow-up
}

Artigo original

Palavras-chave

Metaplasia

Ossificação heterotópica/patologia Endométrio/patologia

Histeroscopia Infertilidade/etiologia

Keywords

Metaplasia

Ossification, heterotopic/patologia

Endometrium/pathology

Hysteroscopy Infertility/etiology

\section{Resumo}

OBJETIVO: apresentar o quadro clínico das pacientes com metaplasia óssea e avaliar os fatores de risco, as mudanças dos sinais e sintomas após a retirada do fragmento ósseo. MÉTODOS: foi realizado um estudo transversal com 16 pacientes diagnosticadas com fragmentos ósseos na cavidade uterina no período de julho de 2006 a janeiro de 2009. O critério de inclusão foi o achado de fragmento ósseo retirado da cavidade uterina. Todas as pacientes tiveram confirmação histológica de presença de tecido ósseo na cavidade endometrial. Obtivemos os dados de todas as pacientes antes e depois da retirada por meio de um questionário para avaliar o efeito da retirada sobre a sintomatologia das pacientes, além da pesquisa de possíveis fatores relacionados ao aparecimento da doença. RESULTADOS: metade das pacientes (8/16) tinha sintomas hemorrágicos e um terço (6/16) apresentava infertilidade. A retirada dos fragmentos foi efetiva na melhora das queixas, havendo desaparecimento dos sintomas em todos os casos de menorragia e dor pélvica. CONCLUSÃO: A retirada do fragmento ósseo pode restaurar a fertilidade em pacientes selecionadas e que tenham como causa a metaplasia óssea, além de ser bastante efetiva em proporcionar melhora nos casos que cursam com dor pélvica e menorragia.

\section{Abstract}

PURPOSE: to describe the clinical signs and symptoms of patients with bone metaplasia and to assess the risk factors for changes in these symptoms after removal of the bone fragment. METHODS: a cross-sectional study was conducted on 16 patients with a diagnosis of bone fragments in the uterine cavity during the period comprising July 2006 to January 2009. The inclusion criterion was the detection of a bone fragment removed from the uterine cavity. The presence of bone tissue in the endometrial cavity was histologically confirmed in all patients. The data of all patients were obtained before and after removal by means of a questionnaire for the evaluation of the effect of removal on the symptoms and for the search of possible factors related to the onset of the disease. RESULTS: half the patients (8/16) had hemorrhagic symptoms and one third (6/16) were infertile. Removal of the fragments was quite effective in improving the complaints, with the disappearance of symptoms in all cases of hemorrhage and of pelvic pain. CONCLUSION: removal of bone fragments can restore the fertility of selected patients whose infertility is caused by bone metaplasia and is quite effective in leading to improvement in patients with pelvic pain and menorrhage.
Correspondência:

Rua Napoleão de Barros, $715,7^{\circ}$ andar CEP 04024-002 - São Paulo (SP), Brasil Fone: (11) 5579-3321 Fax: (11) 5549-2127 E-mail: raphaelcmparente@hotmail.com

Recebido

19/11/09

Aceito com modificacōos
Departamento de Ginecologia da Escola Paulista de Medicina da Universidade Federal de São Paulo - UNIFESP - São Paulo (SP), Brasil. ' Ginecologista do Ministério da Saúde e da Universidade Federal do Rio de Janeiro - UFRJ - Rio de Janeiro (RJ), Brasil.

2 Professor-associado de Ginecologia da Universidade Federal de São Paulo - UNIFESP - São Paulo (SP), Brasil.

${ }_{3}^{3}$ Professor adjunto do Instituto de Biologia da Universidade Federal do Rio de Janeiro - UFRJ - Rio de Janeiro (RJ), Brasil.

${ }_{4}^{4}$ Professor adjunto chefe do Departamento de Ginecologia da Universidade do Estado do Rio de Janeiro - UERJ - Rio de Janeiro (RJ), Brasil.

${ }^{5}$ Professor adjunto do Departamento de Ginecologia da Universidade Federal Fluminense - UFF - Rio de Janeiro (RI), Brasil.

${ }^{6}$ Médica da Universidade Federal de São Paulo - UNIFESP - São Paulo (SP), Brasil.

7 Ginecologista do Hospital dos Servidores do Estado do Rio de Janeiro do Ministério da Saúde - Rio de Janeiro (RJ), Brasil.

Não houve qualquer suporte financeiro.

Não há conflito de interesse. 


\section{Introdução}

A metaplasia óssea do endométrio é caracterizada pela presença de tecido semelhante ao osso dentro da cavidade uterina. É mais frequente em pacientes com infecções genitais recorrentes, alterações menstruais, dor pélvica crônica e antecedentes de abortamento ou infertilidade ${ }^{1}$. A idade mais prevalente da afecção oscila entre 20 e 40 anos $^{2}$, mas já foi descrita na pós-menopausa ${ }^{3}$. Embora a prevalência real desta afecção não seja conhecida, estima-se que alcance 0,15\% dos casos encaminhados para clínicas de histeroscopia, dado embasado em estudo de prevalência ${ }^{4}$.

Apesar de fragmentos ósseos na cavidade uterina serem chamados genericamente de metaplasia óssea, conceitualmente e etimologicamente somente poderiam assim ser classificados aqueles derivados do endométrio, uma vez que metaplasia é a transformação de tecido adulto em outro igualmente adulto ${ }^{5}$. Embora a metaplasia óssea do endométrio tenha etiologia e patogênese controversas, observa-se que mais de $80 \%$ ocorrem após gestações prévias, principalmente nas que resultaram em abortamentos $^{4,6}$. Não obstante, muito se discute sobre a etiologia dessa afecção. Enquanto alguns pesquisadores insistem em afirmar que a presença de restos ovulares é fundamental ${ }^{1}$, outros defendem a existência de metaplasia verdadeira ${ }^{4}$. A maioria, porém, admite a ocorrência das duas vias, originando-se tanto de abortamentos como de metaplasia verdadeira ${ }^{1-3}$, considerando, entretanto, a necessidade de estímulos ligados às modificações gravídicas do organismo materno sobre o endométrio.

Esses fatos ilustram claramente a discordância no tocante à etiologia da metaplasia óssea do endométrio. Entretanto, vale ressaltar que, dentre as teorias mais aceitas, a calcificação e a ossificação distrófica do tecido ovular residual pós-abortamento e a heteroplasia das células estromais uterinas multipotentes latentes são as mais consistentes. Ainda, a metaplasia pode ser diferenciada pela histologia dos fragmentos ósseos oriundos de restos conceptuais pela ausência de reação tecidual ao seu redor, além da falta de ossificação endocondral, já que esse tipo de osteogênese ocorre somente após o primeiro trimestre de gravidez ${ }^{7}$.

Nosso grupo, em estudo recente, demonstrou a etiologia em oito casos, caracterizando a metaplasia os tecidos originários da própria paciente ${ }^{8}$, e não remanescentes de abortamentos. $\mathrm{O}$ objetivo deste artigo foi demonstrar os fatores de risco para a lesão e o quadro clínico das pacientes com metaplasia óssea e avaliar a possível melhora de sinais e sintomas após a retirada do fragmento ósseo.

\section{Métodos}

Foi realizado um estudo no qual foram incluídas pacientes com o diagnóstico de fragmentos ósseos na cavidade uterina no período de julho de 2006 a janeiro de 2009. Devido à raridade da doença, houve busca ativa de qualquer caso suspeito ou confirmado de metaplasia óssea uterina após contato prévio com diversos centros de endoscopia, patologia e imagem do Estado do Rio de Janeiro. Após o aceite da paciente e confirmação do caso como metaplasia óssea verdadeira, eram incluídas no estudo e atendidas para responderem ao questionário e realizarem coleta de sangue no Hospital Universitário Pedro Ernesto. O local de retirada dos fragmentos foi o próprio centro inicial de atendimento da paciente, seja privado ou público. Nos casos novos, havia a presença do pesquisador principal para coletar imediatamente os fragmentos ósseos para análise histopatológica e genética. O critério de inclusão foi o achado de fragmento ósseo retirado da cavidade uterina. Todas as pacientes tiveram confirmação histológica da presença de tecido ósseo na cavidade endometrial, sendo descartadas aquelas que tinham o diagnóstico de calcificação apenas. O critério de exclusão era a discordância em participar do estudo. O estudo foi previamente submetido a e aprovado pelo Comitê de Ética em Pesquisa do Hospital Universitário Pedro Ernesto da Universidade do Estado do Rio de Janeiro. Todas as pacientes assinaram o termo de consentimento livre e esclarecido.

Neste período, foi possível selecionar 16 pacientes para participarem do estudo. O nosso objetivo primário foi avaliar a etiologia do material ósseo por meio da comparação de DNA. Nesta investigação, obtivemos os dados de todas as pacientes antes e depois da retirada, além da pesquisa de possíveis fatores relacionados ao aparecimento da doença. Para pesquisa de dados referentes a sinais, doenças e sintomas prévios ao diagnóstico e retirada dos fragmentos ósseos e após o procedimento de extração, utilizou-se um questionário. A forma de retirada, assim como os antecedentes obstétricos, também foi avaliada por meio deste questionário. $\mathrm{O}$ diagnóstico foi dado sempre por meio do resultado histopatológico de tecido ósseo. Estes fragmentos foram retirados por meio de histeroscopia após visualização de fragmentos semelhantes a ossículos. $\mathrm{Na}$ maioria dos casos, a histeroscopia foi solicitada por suspeita ultrassonográfica.

Resumidamente, para cada paciente solicitou-se pessoalmente, através do pesquisador principal: idade atual, idade na descoberta da presença do fragmento ósseo, idade na época da retirada do fragmento ósseo, meio pelo qual foi efetuada a retirada (curetagem, histeroscopia cirúrgica ou ambulatorial, etc.). Foi incluído na investigação o método pelo qual foi feito o diagnóstico imagenológico que levou à indicação da retirada (histeroscopia, ultrassonografia (Figuras 1 e 2) ou ambos, sinais e sintomas antes e depois da retirada, história gestacional pregressa (partos e abortos), tempo decorrido entre o último aborto (caso tenha ocorrido) e a descoberta do fragmento ósseo e, por último, doenças concomitantes. 


\section{Resultados}

Os resultados são apresentados nas Tabelas 1 e 2 . A média de idade no diagnóstico da metaplasia óssea foi de 40,4 anos. Na nossa série de casos houve uma grande heterogeneidade quanto à faixa etária das pacientes, variando de 20 a 67 anos. A variação de tempo entre o último abortamento e o diagnóstico foi de 4 meses a 40 anos (Tabela 1). Avaliando-se clinicamente todas as pacientes inicialmente alocadas para essa pesquisa, foi observada alta incidência de menorragia. Metade das pacientes de nossa casuística referia, no momento do diagnóstico, queixas relacionadas a alterações no fluxo menstrual, mais especificamente de menorragia. Não houve queixa de irregularidade menstrual (metrorragia). Quatro pacientes eram assintomáticas no momento do diagnóstico definitivo, tendo sido a suspeita levantada por ultrassonografia de rotina. Há, também, relatos de pacientes que foram surpreendidas com a saída de fragmentos ósseos junto com o fluxo catamenial. A dor pélvica esteve presente em 25\% das pacientes no momento do diagnóstico, sendo que em metade das vezes era do tipo dismenorreia. A infertilidade, comumente citada nos relatos de casos que versam sobre metaplasia óssea, é um dos achados mais prevalentes e foi encontrado em pouco mais de um terço delas. Houve uma paciente que, após abortamento provocado, foi submetida à laparotomia exploradora e seis cirurgias subsequentes por complicações sépticas. Outra paciente teve gravidez tubária tratada com salpingectomia unilateral. Esses dois casos ilustram situações em que houve prejuízo do processo reprodutivo, sem que a metaplasia óssea fosse a única causa determinante da infertilidade. Por outro lado, não foi observado nenhum caso de doença sistêmica, como o hiperparatireoidismo.

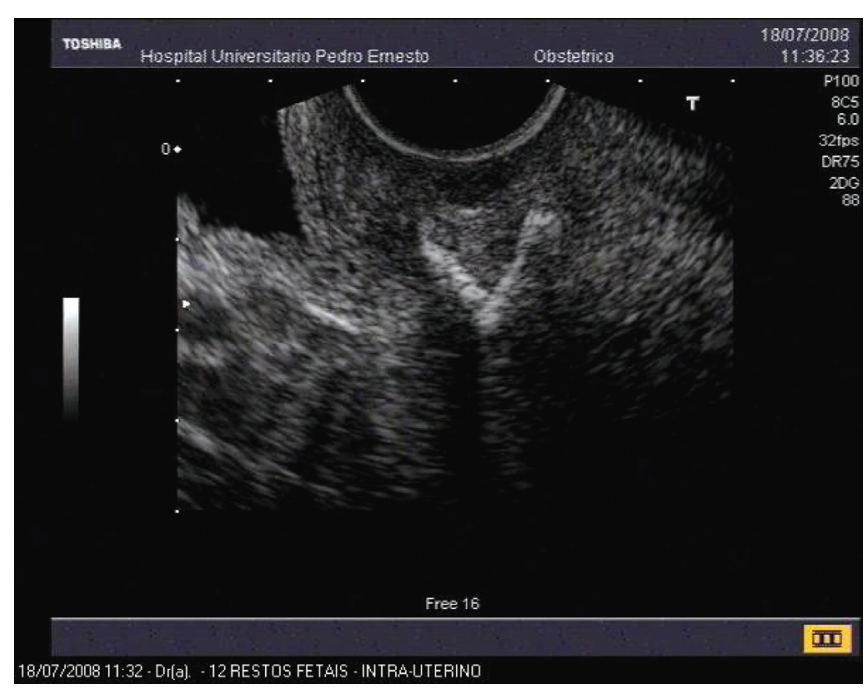

Figura 1 - Imagem ultrassonográfica da metaplasia óssea do endométrio. Observar área hiperecogênica na cavidade uterina. Embora o aspecto seja sugestivo de calcificação de restos ovulares ou DIU, foi confirmada a origem endometrial por meio da comparação do DNA.

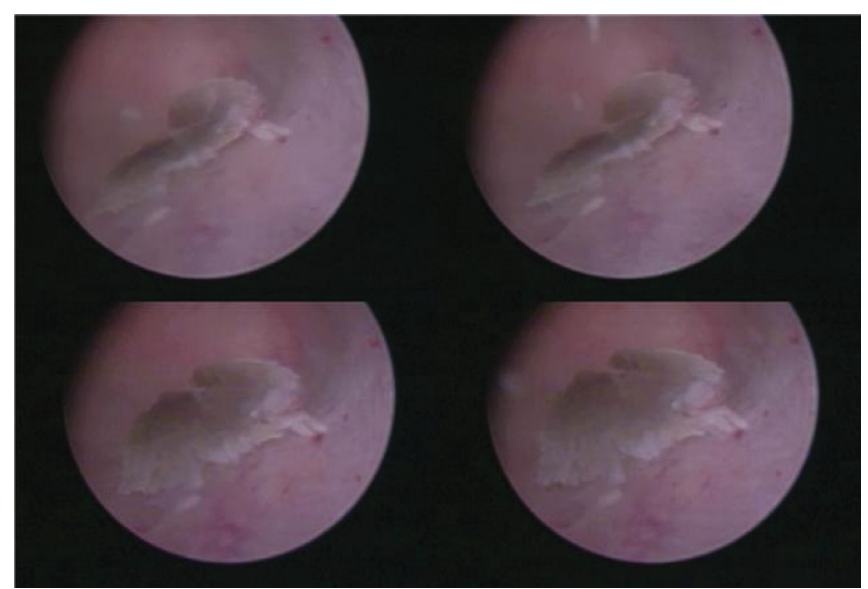

Figura 2 - Aspecto histeroscópico da metaplasia óssea. Os fragmentos são irregulares e muito espiculados.

Tabela 1 - Antecedentes obstétricos em 14 casos com metaplasia óssea endometrial

\begin{tabular}{|c|c|c|c|c|c|c|}
\hline Paciente & Idade (anos) & Gestações (n) & Paridade (n) & $\begin{array}{l}\text { Tempo desde último } \\
\text { abortamento (anos) }\end{array}$ & Tipo de abortamento & Procedimento \\
\hline 1 & 39 & 1 & 0 & 10 & Induzido & Curetagem \\
\hline 2 & 30 & 1 & 0 & 3 & Tubário & Laparotomia \\
\hline 3 & 54 & 8 & 5 & 16 & Induzido & Curetagem \\
\hline 4 & 46 & 1 & 0 & 24 & Induzido & Curetagem \\
\hline 5 & 28 & 1 & 0 & 1 & Espontâneo & Curetagem \\
\hline 6 & 42 & 6 & 5 & 10 & Induzido & Curetagem \\
\hline 7 & 28 & 2 & 1 & 10 & Espontâneo & Espontâneo \\
\hline 8 & 37 & 3 & 2 & 12 & Espontâneo & Curetagem \\
\hline 9 & 29 & 3 & 0 & 2 & Espontâneo & Espontâneo \\
\hline 10 & 47 & 6 & 3 & 19 & Espontâneo & Curetagem \\
\hline 11 & 53 & 5 & 1 & 40 & Espontâneo & Curetagem \\
\hline 12 & 60 & 3 & 2 & 26 & Induzido & Curetagem \\
\hline 13 & 20 & 1 & 0 & 2 & Induzido & Curetagem \\
\hline 14 & 67 & 4 & 1 & 40 & Espontâneo & Espontâneo \\
\hline Mediana (variação) & $40,5(20-67)$ & $3(1-8)$ & $1(0-5)$ & $11(1-40)$ & & \\
\hline
\end{tabular}

CC: Centro Cirúrgico. As pacientes 10 e 12 queixavam-se de saída de fragmentos ósseos pela vagina. 
Tabela 2 - Queixa das pacientes com diagnóstico clínico de metaplasia óssea e exclusão por retirada

\begin{tabular}{|c|c|c|c|c|c|}
\hline Idade atual & $\begin{array}{c}\text { Sangramento uferino } \\
\text { anormal }\end{array}$ & Dor & Infertilidade & $\begin{array}{l}\text { Idade na época do } \\
\text { diagnóstico }\end{array}$ & Melhora após a retirada \\
\hline 52 & Sim & Não & Não & 47 & Sim \\
\hline 29 & Sim & Não & Não & 29 & Sim \\
\hline 39 & Não & Sim & Sim & 39 & $\begin{array}{c}\operatorname{Sim}^{*} \\
\text { Não tentou gestar }\end{array}$ \\
\hline 46 & Sim & Sim & Sim & 46 & $\begin{array}{l}\text { Sim para sangramento e dor. } \\
\text { Permaneceu infértil }\end{array}$ \\
\hline 59 & Não & Não & Não & 40 & AS \\
\hline 74 & Não & Não & Não & 70 & AS \\
\hline 63 & Não & Não & Não & 60 & AS \\
\hline 42 & Sim & Não & Sim & 42 & $\begin{array}{l}\text { Sim para sangramento e dor. } \\
\text { Permaneceu infértil }\end{array}$ \\
\hline 38 & Sim & Não & Não & 38 & Sim \\
\hline 26 & Sim & Sim & Não & 26 & Sim \\
\hline 28 & Não & Não & Sim & 28 & Sim \\
\hline 41 & Não & Não & Não & 41 & AS \\
\hline
\end{tabular}

AS: assintomática.

\# houve melhora dos sintomas; a fertilidade não pôde ser avaliada, pois a paciente não tentou uma gravidez.

Muito consistente foi a melhora da sintomatologia após a retirada dos fragmentos, sobremaneira os hemorrágicos e os álgicos. Das nove pacientes que apresentavam pelo menos um destes sintomas, somente uma teve melhora parcial, e as demais relataram recuperação completa (Tabela 2). De seis pacientes que se queixavam de infertilidade, duas engravidaram posteriormente à retirada dos fragmentos. Além disso, duas pacientes já estavam em idade com possibilidade menor de gravidez (entre 42 e 46 anos), e outra desistiu de participar do seguimento. A sexta paciente ainda tentava engravidar na época da redação deste artigo. A história de aborto é universal e não há diferença entre o provocado e o espontâneo. Não tivemos nenhum caso de nuliparidade, embora uma paciente tenha tido somente uma história de gravidez ectópica e nenhum relato de abortamento intrauterino prévio. Finalmente, o achado menos observado em nossas pacientes foi a eliminação de "pó branco" ou de "pedaços de ossos" pela vagina (Tabelas 2). A Tabela 2 mostra as queixas e o relato de melhora. No caso da infertilidade, somente foi classificado como 'sem melhora' a paciente que tentou engravidar e não conseguiu, o que não ocorreu com toda a amostra, impossibilitando avaliar o real papel da retirada no tratamento.

\section{Discussão}

Esta é a maior casuística em âmbito mundial sobre sinais e sintomas de pacientes com metaplasia óssea endometrial. Até então, a maior série de casos, publicada em 2009, incluiu cinco pacientes?.

A metaplasia óssea cursa, em grande parte, com irregularidade menstrual, dor pélvica crônica, dispareunia, corrimento vaginal e infertilidade ${ }^{4}$. Pode ser, ainda, assintomática ${ }^{1}$. Há interessantes relatos de pacientes que foram surpreendidas com a saída de fragmentos ósseos junto com o fluxo menstrual ${ }^{10,11}$, o que também foi encontrado em nosso estudo. Avaliando-se clinicamente todas as pacientes inicialmente alocadas para essa pesquisa, conseguimos resultados que, em parte, são concordantes com a literatura, nos quais se observa alta incidência de queixas relacionadas a sangramento anormal. Por outro lado, não foi verificado nenhum caso de hiperparatireoidismo, o que de certa forma diverge da literatura pertinente. Como limitante para a validade deste último resultado, temos que ressaltar que não foi feito qualquer exame laboratorial para excluir ou confirmar essa ou outra doença que pudesse alterar o metabolismo do cálcio. Somente aplicamos um questionário para as participantes do estudo questionando a existência dessas doenças.

Destas 16 pacientes, foi possível extrair DNA ósseo e sanguíneo de oito pacientes. Em todas estas, os fragmentos ósseos têm origem metaplásica, já que o perfil genético de células do sangue periférico foi o mesmo do fragmento ${ }^{8}$. Embora todas tivessem história de abortamento (até o terceiro mês), nenhuma diferença foi detectada no DNA, o que seria esperado caso o tecido ósseo fosse proveniente de restos ovulares. Este resultado é inesperado, já que é totalmente diferente da proporção estimada pela literatura 
prévia baseada especificamente na epidemiologia dos casos sem análise genética. Estes achados reforçam a teoria de alguns autores de que até o terceiro mês não é esperada formação óssea fetal. É possível que células totipotentes fetais tenham estimulado a transformação do endométrio em tecido semelhante ao osso (metaplasia).

Entre as teorias mais bem aceitas para se explicar a etiologia da metaplasia óssea endometrial, estão as da calcificação distrófica (incrustação de sais em tecidos previamente lesados, com processos regressivos ou necrose), a da calcificação de restos ovulares e das células totipotentes presentes no endométrio e com capacidade de se transformarem em tecido ósseo ou cartilaginoso ${ }^{12}$. Algumas desordens metabólicas, como hipercalcemia, hipervitaminose $\mathrm{D}$, hipofosfatemia e hiperparatireoidismo, podem ser associadas ao achado de fragmentos ósseos no interior da cavidade uterina ${ }^{13}$. Além disso, a estimulação prolongada do endométrio por estrogênios sem a contraposição da progesterona também é considerada fator promotor de metaplasia óssea endometrial ${ }^{14}$. Em nosso estudo, o único fator associado com o achado de metaplasia do endométrio dentro da cavidade uterina foi história prévia de abortamento. Todas as pacientes foram questionadas em relação aos outros fatores de risco e não houve nenhum relato deles.

Esses sintomas e sinais descritos decorrem, em sua maioria, da endometrite reacional consequente à presença da afecção na cavidade uterina, muito semelhante ao ocorrido na mucosa endometrial na presença de um dispositivo intrauterino, alterações que também dificultam a nidação do blastocisto, e por isso esse método contraceptivo é considerado abortivo ${ }^{15}$.
O diagnóstico da metaplasia óssea do endométrio pode ser feito por meio de exame ultrassonográfico pélvico ou transvaginal que poderá evidenciar área hiperecogênica na cavidade uterina, ou por meio da histeroscopia, pela visualização de lâmina esbranquiçada com aspecto que lembra osso, calcificação ou tecido fibroso, ou pela própria visualização direta do fragmento. $\mathrm{O}$ melhor momento para realização da endoscopia é durante o início da fase proliferativa do ciclo menstrual, ocasião em que a menor espessura do endométrio facilita a inspeção de toda a cavidade do útero.

O tratamento da metaplasia óssea é realizado por meio de retirada histeroscópica dos fragmentos ósseos ou por curetagem uterina ${ }^{16}$. Com a retirada dos fragmentos ósseos, há diminuição da secreção local de prostaglandinas, e o volume do fluxo menstrual é reduzido em cerca de $50 \%{ }^{15}$. Além disso, restaura-se a fertilidade, obviamente se não existirem outras afecções concomitantes ${ }^{11,16-18}$.

Em resumo, a metaplasia óssea uterina geralmente cursa com aumento do fluxo menstrual e pode ser causa de infertilidade, embora não sejam raras situações em que a paciente é assintomática e somente descobre o fragmento ósseo acidentalmente, durante um exame ultrassonográfico de rotina. Em pacientes sintomáticas, o tratamento é bastante eficiente na resolução das queixas e deve sempre ser aventado preferencialmente por via histeroscópica. A retirada do fragmento ósseo pode restaurar a fertilidade em pacientes selecionadas e que tenham como causa a metaplasia óssea, e é bastante efetiva em proporcionar melhora nos casos que cursam com dor pélvica e menorragia.

\section{Referências}

1. Tulandi T, Al-Sunaidi M, Arseneau J, Tonin PN, Arcand SL. Calcified tissue of fetal origin in utero. Fertil Steril. 2008;89(1):217-8.

2. Shimizu $M$, Nakayama $M$. Endometrial ossification in a postmenopausal woman. J Clin Pathol. 1997;50(2):171-2.

3. Grigoras D, Vasile L, Chiriac VD, Mazilu O, Qatawneh-Gavrilita B, Citu C. Osseous metaplasia of the uterus. Timisoara Med J. 2003;53(3-4):283-5.

4. Makris N, Stefanidis K, Loutradis D, Anastasiadou K, Hatjipappas $G$, Antsaklis $A$. The incidence of retained fetal bones revealed in 2000 diagnostic hysteroscopies. JSLS. 2006;10(1):76-7.

5. Slack JM. Epithelial metaplasia and the second anatomy. Lancet. 1986;2(8501):268-71

6. Melius F, Julian TM, Nagel TC. Prolonged retention of intrauterine bones. Obstet Gynecol. 1991;78(5 Pt 2):919-21.

7. Bhatia NN, Hoshiko MG. Uterine osseous metaplasia. Obstet Gynecol. 1982;60(2):256-9.

8. Parente RCM, Patriarca MT, de Moura Neto RS, de Oliveira MAP, Lasmar RB, de Holanda Mendes P, et al. Genetic analysis of the cause of endometrial osseous metaplasia. Obstet Gynecol. $2009 ; 114(5): 1103-8$.

9. Lousquy R, Deffieux X, Gervaise A, Faivre E, Frydman R, Fernandez $H$. Fertility after hysteroscopic management of osseous metaplasia of the endometrium. Int J Gynaecol Obstet. 2009; 106(3): 254-5

10. Basu M, Mammen C, Owen E. Bony fragments in the uterus: an association with secondary subfertility. Ultrasound Obstet Gynecol. $2003 ; 22(4): 402-6$.

11. Srofenyoh K, Addison M, Dortey B, Kuffour P. Intrauterine retained fetal bones as a cause of secondary infertility. Ghana Med J. 2006;40(3): 105-9.

12. Wolff EF, Wolff $A B$, Hongling DU, Taylor HS. Demonstration of multipotent stem cells in the adult human endometrium by in vitro chondrogenesis. Reprod Sci. 2007;14(6):524-33.

13. Van den Bosch T, Van Schoubroeck D, Timmerman D, Deprest J. Uterine intramural bone after mid-trimester termination of pregnancy may not affect fertility: a case report. Ultrasound Obstet Gynecol. $2003 ; 22(4): 407-8$. 
14. Bedaiwy MA, Goldberg JM, Biscotti CV. Recurrent osseous metaplasia of the cervix after loop electrosurgical excision. Obstet Gynecol. 2001;98(5 P+ 2):968-70.

15. Lewis V, Khan-Dawood F, King M, Beckmann C, Dawood MY. Retention of fetal bone increases menstrual prostaglandins. Obstet Gynecol. 1990;75(3 Pt 2):561-3.

16. Acharya U, Pinion SB, Parkin DE, Hamilton MP. Osseous metaplasia of the endometrium treated by hysteroscopic resection. $\mathrm{Br}$ J Obstet Gynaecol. 1993;100(4): 391-2.

17. Bahceci M, Demirel LC. Osseous metaplasia of the endometrium: a rare cause of infertility and its hysteroscopic management. Hum Reprod. 1996; 11 (11):2537-9.

18. Onderoglu LS, Yarali H, Gultekin M, Katlan D. Endometrial osseous metaplasia: an evolving cause of secondary infertility. Fertil Steril. 2008;90(5):2013.e9-11. 